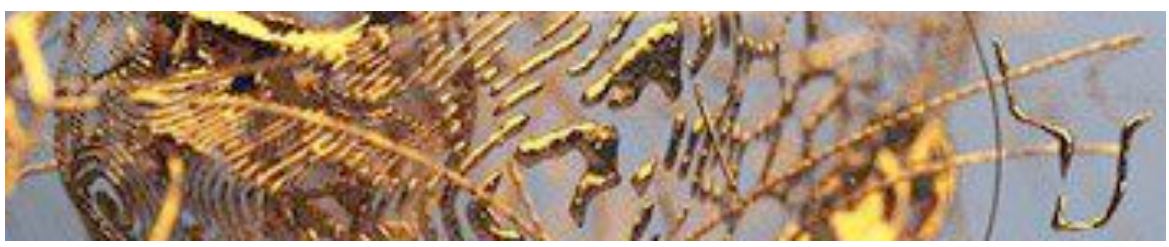

\title{
Tratamiento periodístico del Movimiento 15-M en medios de comunicación alternativos y convencionales
}

\section{Press coverage of Movimiento 15- $M$ in alternative and conventional media}

\author{
Moreno Ramos, Miguel Ángel ${ }^{(*)}$ \\ Universidad Carlos III de Madrid - España \\ miguelangelmramos@telefonica.net
}

\begin{abstract}
Resumen
El surgimiento del Movimiento 15-M como hecho informativo durante el mes de mayo de 2011 provocó la organización de coberturas por parte de los medios de comunicación que revelaron diferencias en los enfoques entre los medios de carácter alternativo y convencional. Tras un análisis de contenido de los artículos de distintos medios convencionales y alternativos, complementado con entrevistas a periodistas de cada uno de los medios analizados, se han hallado algunas diferencias que retratan una cobertura más innovadora y centrada en los protagonistas del movimiento en los medios alterna-
\end{abstract}

\begin{abstract}
The rising of 'Movimiento 15- $M$ ' in May 2011 as an informative fact caused the assumption of different strategies in media's coverage that showed many differences in framing between alternative media, and conventional media. Due to a content analysis completed with interviews to each media's journalists, we have found many differences between alternative media's coverage, more innovative and closer to the activists, and conventional media's news, more linked to political consequences of this social movement.
\end{abstract}


Miguel Ángel Moreno Ramos

«Tratamiento periodístico del Movimiento 15-M en medios de comunicación alternativos...»

tivos, frente al tratamiento más orientado a la repercusión política del acontecimiento por parte de los medios convencionales.

Palabras clave: periodismo, Movimiento 15-M, medios alternativos, moviKeywords: journalism, movimiento 15lizaciones sociales. 
«Tratamiento periodístico del Movimiento 15-M en medios de comunicación alternativos...»

\section{INTRODUCCIÓN}

Esta investigación ofrece un acercamiento teórico a la cobertura de las movilizaciones sociales producidas a partir del día 15 de mayo de 2011 en varias ciudades españolas, especialmente entre las diferencias en el encuadre informativo efectuado por medios de carácter alternativo y medios tradicionales, entendidas ambas categorías como muestra de dos tipos de organización periodística diferenciables entre sí a través del sus características, financiación o enfoque frente a la actualidad.

Los sucesos informativos que fundamentan el estudio son las protestas ciudadanas sucedidas a partir de la manifestación convocada por la plataforma Democracia Real Ya el 15 de mayo del 2011 en Madrid y otras ciudades, que en la capital española desemboca en una acampada en la céntrica Puerta del Sol, alrededor de la cual se gesta un movimiento reivindicativo y asambleario que sobrepasa el mes de duración y atraviesa unas elecciones municipales en todo el país, sucedidas el 22 de mayo del mismo año. Dichas movilizaciones y acampadas se producen también en varias ciudades españolas, europeas y latinoamericanas.

Entre los objetivos de la investigación no está el de teorizar respecto a las causas, acontecimientos y consecuencias de esta ola de reivindicaciones, identificada a nivel general como "Movimiento 15-M", sino el de aproximarse a ellas desde el punto de vista comunicativo y periodístico.

De esta forma, en este estudio ahondamos en dos inquietudes distintas: la de analizar la cobertura de un acontecimiento informativo en la frontera entre lo social y lo político y el objetivo de encontrar diferencias en la forma de encarar dicha cobertura por dos tipos de instancias periodísticas opuestas en cuanto a su organización, rutinas productivas o financiación.

Para esta aproximación a dos tipologías de organización periodística se han seleccionado dos medios de carácter alternativo en su versión en la Red, que son Periodismo Humano (http://www.periodismohumano.com) y Diagonal (http://www.diagonalperiodico.net). Se trata de dos medios periodísticos relacionados o con especial sensibilidad hacia el ámbito asociativo, la defensa de los derechos humanos o la movilización social; que además se destacaron como fuentes informativas de primer nivel desde un ámbito no convencional, especialmente en el caso de Periodismo Humano, que fue citado por medios internacionales por su cobertura de las movilizaciones. 
«Tratamiento periodístico del Movimiento 15-M en medios de comunicación alternativos...»

En cuanto a los medios tradicionales, los escogidos han sido El País (http://www.elpais.com) y Público (http://www.publico.es), dos empresas periodísticas con una organización convencional, cuya tendencia editorial permite hacer un análisis más centrado en los enfoques periodísticos que en las diferencias ideológicas.

El estudio ha requerido la búsqueda y selección de artículos publicados en las ediciones digitales de los cuatro medios entre los días 15 y 30 de mayo de 2011, de los cuales ha surgido una selección de 461 piezas periodísticas en total. Estos documentos han sido tratados por medio de una ficha de análisis cualitativo, en la que se han incluido clasificaciones de encuadre utilizadas internacionalmente, así como otros datos de estilo periodístico y análisis de fuentes. Como complemento se han realizado entrevistas en profundidad con cuestionario abierto a profesionales de los cuatro medios, de manera que se han podido contrastar algunos de los resultados con sus opiniones acerca de la cobertura realizada.

Dos cuestiones han guiado esta investigación: ¿Cuál ha sido la cobertura del conocido como "Movimiento 15-M" a partir del análisis de los artículos escritos entre los días 15 y 30 de mayo en dos medios periodísticos convencionales como El País y Público, en comparación con dos medios alternativos como Diagonal y Periodismo Humano, todos ellos en sus versiones en la Red?, y ¿qué diferencias se observan entre los enfoques informativos sobre las manifestaciones del 15 de mayo entre los medios de carácter alternativo y los medios convencionales en los artículos publicados en sus versiones web?

Los objetivos del estudio podrían definirse en dos planos. En primer lugar, se estableció como meta primaria la observación de la presencia de estas movilizaciones en las agendas informativas de los cuatro medios sometidos a estudio. En segundo término, se estudiaron esas diferencias por medio de un análisis de contenido bajo el paradigma del estudio de encuadre o framing.

Las hipótesis previas del estudio incluyeron la percepción de que las cabeceras alternativas otorgan mayor importancia a los temas de alcance social en la composición de su agenda, mientras que los medios tradicionales tratan más los asuntos de tipo político y declarativo. También se pronosticó un tratamiento más local por parte de los medios alternativos y una 
«Tratamiento periodístico del Movimiento 15-M en medios de comunicación alternativos...»

mayor utilización de portavoces sociales como fuentes, frente a la posible recurrencia a las fuentes políticas por parte de los medios tradicionales.

Entre los motivos que justificaron el estudio se valoraron tanto la oportunidad de indagar en las diferencias entre dos organizaciones periodísticas a nivel general como el de observar estas distinciones en el ámbito concreto del 15-M, un acontecimiento social que marcó la agenda informativa de unos y otros durante más de un mes, aunque en este caso la investigación se centró en quince días, entre la primera manifestación (15 de mayo de 2011) y el día 31 del mismo mes.

\section{MARCo Té́rico}

En el ámbito teórico, es necesario dibujar en primer lugar una línea entre los medios alternativos y convencionales. Comenzaremos por la noción de medio alternativo, en la que incluimos a Periodismo Humano y Diagonal.

Se trata de una categoría basada en una línea de investigación presente en la tradición anglosajona a partir de autores como el norteamericano John Downing, que acuña la noción de "medios radicales" o el británico Chris Atton, que trabaja sobre los medios alternativos desde una perspectiva amplia y cultural; pero también explorada ampliamente en América Latina.

Para Downing, los medios radicales son aquellos "políticamente disidentes" que ofrecen "alternativas radicales al debate público" (1990: 181) y se relacionan con una noción de "audiencia activa", que no solo consume contenidos informativos, sino que también es sujeto de creación.

Atton destaca en su visión de los "medios alternativos" otras características como la independencia comercial y editorial, la libertad periodística y el "otorgamiento de poder a grupos de interés específicos" (2002: 13); pero también realiza una ampliación del campo de estudio, hablando no tanto de medios de comunicación sino de un "área de producción cultural" alternativa. Este pensador focaliza su interés en la capacidad de estos medios para encontrar métodos de creación, producción o distribución no convencionales, por encima de su contenido informativo, ya que valora estos medios por su capacidad para dar voz a grupos de opinión excluidos por la comunicación tradicional. 
«Tratamiento periodístico del Movimiento 15-M en medios de comunicación alternativos...»

En este contexto, podemos definir a los medios alternativos como aquellos que "rechazan la política institucional en el sentido de que persiguen un cambio en la sociedad o al menos una revisión crítica de los valores tradicionales" (O’Sullivan, 1994, en Atton, 2002:15) una visión que recoge Atton, quien también retoma las nociones del alemán Hans Magnus Enzensberger, acerca del "uso emancipador de los medios" como precursor de esta relación más estrecha entre el medio y su público que hemos señalado con anterioridad.

El concepto de medios alternativos también tiene su eco en pensadores de la comunicación latinoamericanos, como la colombiana Clemencia Rodríguez (2001), que introduce un nuevo término, el de "medios ciudadanos", para definir aquellos medios que dan poder a las personas para definirse a sí mismas "irrumpiendo en la aceptación pasiva de identidades impuestas por sujetos externos” (Rodríguez, 2009: 17).

Otras aportaciones latinoamericanas de relevancia a esta discusión son las del argentino Mario Kaplún (1985), que utiliza la noción de "medios populares" desde el punto de vista de la educación y la toma de conciencia, relacionada con el pensamiento del pedagogo brasileño Paulo Freire; o la del boliviano Alfonso Gumucio-Dagron, que incide en el aspecto de la participación popular y el uso de los medios de comunicación como herramienta de desarrollo.

Por otra parte, también resulta adecuado para el estudio de los medios alternativos mencionar el concepto del "estatuto de objetividad", enunciado por Hackett y Zhao (1998, en Hamilton y Atton, 2008) como un contenido clave para entender la razón de ser de estos medios y su oposición respecto a los convencionales.

Este concepto pone en duda cuál es el valor primordial de las noticias en un medio informativo: si para los medios tradicionales sería la factualidad de la información -su valor como hecho, lo que conlleva asumir que existe la posibilidad de identificar los datos que contiene con precisión y sin prejuicios-, para un medio alternativo el valor de una información parte de una base distinta: la de los valores. Para este tipo de medios el ejercicio del reporterismo está indisolublemente unido a los valores (personales, profesionales, institucionales), ya que "no es posible separar los hechos de los valores" (Hackett y Zhao, 1998, en Hamilton y Atton, 2008: 127). 
«Tratamiento periodístico del Movimiento 15-M en medios de comunicación alternativos...»

De esta manera, entendemos Diagonal y Periodismo Humano como dos medios que se ajustan a esta definición por su enfoque informativo desde el punto de vista de los valores, algo que confirmó uno de los redactores de Periodismo Humano, Juan Luis Sánchez, que definió la labor de su medio como de periodismo "con un enfoque de Derechos Humanos" (Sánchez, Juan Luis. Entrevista por teléfono el 1 de septiembre de 2011). Por otra parte, su carácter independiente del ámbito comercial, su tendencia a no cubrir información específicamente política y su ocupación hacia comunidades locales, como el caso de Diagonal en sus distintas secciones locales, son otros de los rasgos que sostienen su inclusión en este área.

En cuanto a los medios convencionales, la distinción primordial que nos permite separarlos de las organizaciones periodísticas que hemos caracterizado como alternativas es su carácter empresarial, entendiendo a la empresa informativa como aquella "cuya principal actividad es la producción y comercialización de información periodística, plasmada en un soporte" (Galán, 2008: 19). Sin embargo, también merece la pena recordar su mayor compromiso con el carácter "factual" de las noticias, dentro de la teoría de Hackett y Zhao (1998, en Hamilton y Atton, 2008), en oposición con el compromiso con los valores que éstas transmiten, hacia el que se orientan los medios alternativos.

Es característico de los medios convencionales su papel en la configuración de la agenda setting de la sociedad (Gomis, 1991), los temas que están en el debate público, debido a su posición hegemónica a niveles de audiencia, vinculada también a ese mayor poder para llegar al público debido a la organización empresarial que contienen, que les permite el acceso a canales de distribución y promoción a los que los alternativos no tienen acceso.

Sin entrar en disquisiciones de tipo ideológico, entendemos que tanto El País como Público se ajustan a esta definición de medios convencionales que cuentan con estructura empresarial y forman parte de la configuración de la agenda pública, además de que cuentan con una difusión en todo el país que les permite mantener esa influencia y de una gran presencia en la Red, que es el ámbito en el que se inscribe el análisis.

El criterio para escoger, entre los distintos medios alternativos y convencionales que en España han realizado una cobertura en la Red del Movimiento 15-M, estos cuatro medios para el análisis de contenido, refiere a varias condiciones distintas.

Mediaciones Sociales, № 12, 2013, pp. 160-187. ISSN electrónico: 1989-0494. DOI: http://dx.doi.org/10.5209/rev_MESO.2013.n12.45267 
«Tratamiento periodístico del Movimiento 15-M en medios de comunicación alternativos...»

En primer lugar, en el ámbito de los medios alternativos, la elección de Diagonal viene dada por el propósito inicial del estudio, que no era otro que un trabajo más enfocado en el largo plazo y hacia un estudio de contenidos en medios alternativos de carácter impreso. De esta manera, Diagonal iba a ser contrastado como periódico quincenal con otros medios de características similares en España, como el semanario catalán Setmanari Directa y Novas da Galiza, periódico gallego de carácter mensual.

Sin embargo, la emergencia del 15-M llevó a replantear todo el análisis y centrarse solo en aquellos medios que hicieran un trabajo diario en su web, cosa que no se cumplía en los medios catalán y gallego, pero sí en Diagonal. A esto se unió la gran relevancia que obtuvieron con las protestas otros medios alternativos como Periodismo Humano, que habían realizado coberturas de gran profundidad e innovación, citadas incluso en medios internacionales, lo que llevó a incorporar este medio a la investigación.

Con esta elección se decidió dejar aparte a otros medios alternativos con una alta actividad durante el 15-M, como los portales españoles de Indymedia, la web Kaos en la Red y otros medios. Las razones para no utilizarlos tenían que ver con su gran diferencia en cuanto a estilo, publicación y otros parámetros que los alejaban mucho tanto de El País y Público como de los propios medios alternativos elegidos, Periodismo Humano y Diagonal, más periodísticos en su estilo y forma de publicación.

La decisión de oponer a los medios alternativos en análisis el mismo número de medios convencionales era algo ya cerrado antes del 15-M por motivos de tiempo y posibilidades de análisis, pero la dificultad cabía en la decisión de qué medios podrían ser los más adecuados para identificar las diferencias entre medios convencionales y alternativos y no prestarse a otros análisis de tipo ideológico que no tenían gran interés para el estudio.

Es por esto que, desde el punto de vista de la investigación la elección de El País y Público suponía una opción mejor que el uso de otros medios que también realizaron una extensa cobertura del 15-M desde sus páginas web -como $E l$ Mundo, ABC, La Información, etc.-, debido a que suponían una conveniente eliminación de "ruido" mediático, en el sentido en que estos dos medios son los que más se aproximan ideológicamente a Periodismo Humano y Diagonal, enfocando esta proximidad desde la noción de reformismo, o incluso de izquierda, relacionada con la noción de medio 
«Tratamiento periodístico del Movimiento 15-M en medios de comunicación alternativos...»

alternativo como aquel que está "primordialmente vinculado al cambio social" (Atton 2002, 9).

Desde este planteamiento, analizar Periodismo Humano y Diagonal frente a otros medios convencionales, como pudieran ser, por ejemplo, $A B C$ y La Razón, daría lugar a unas diferencias de enfoque ideológico muy fáciles de adivinar previamente que no iban a ofrecer hallazgos interesantes en el ámbito de las diferencias de enfoques motivada por dualidad convencional-alternativo, que es el objetivo de la investigación.

Partiendo de teorías como las del distanciamiento en Filosofía Política, recogida por el español Cándido Monzón (2006: 251), que rechaza que un mayor número de medios de comunicación den lugar a una mayor comprensión del entorno, sino que apunta a que la mayor cantidad de medios provoca una dispersión de la opinión pública en distintas "opiniones públicas", ya que los ciudadanos no están "homogéneamente bien informados" de inmediato por el mero hecho de que existan muchos medios de comunicación, sino otros factores no necesariamente vinculados a esa pluralidad de medios como el nivel de estudios o el nivel socioeconómico provocan el distanciamiento entre estas distintas opiniones públicas.

La necesidad de no confrontar "opiniones públicas" sino distintas formas de hacer periodismo desde el punto de vista organizativo y laboral de los medios es la que nos lleva a aislar, al menos para este análisis, a medios de otras tendencias ideológicas, para mantener a medios que consideramos medianamente homologables para hacer un buen análisis de contenido centrado exclusivamente en lo periodístico de su contenido.

El estudio de los medios alternativos desde el punto de vista de su producción periodística es un ámbito de la investigación académica que muestra un particular vacío, no solo en España sino también en el contexto europeo, en el que se pueden encontrar muy pocos ejemplos recientes.

Entre ellos está el estudio realizado por la investigadora griega Dimitra Milioni respecto a la página local de Atenas del medio alternativo Indymedia, que analizó durante un periodo de 10 días -entre el 1 y el 10 de noviembre de 2002-, examinando tanto el modelo de periodismo que se hace en sus artículos como en la función del sitio como foro de intercambio de opiniones, creación de comunidades, la relación entre lo local y lo global, además de su vinculación con los movimientos sociales del entorno (Milioni, 2009: 414). 
«Tratamiento periodístico del Movimiento 15-M en medios de comunicación alternativos...»

En el caso español, el único ejemplo de estudio de caso que hemos podido rastrear es el realizado por Jeff Juris sobre la sede de Indymedia en Barcelona entre julio de 2001 y agosto de 2002, recogido en el volumen colectivo La red es de todos (coordinado por Víctor Marí Sáez).

Indymedia es un ejemplo muy claro de medio alternativo o contrahegemónico que surge durante las protestas contra la reunión de la Organización Mundial del Comercio (OMC) en la ciudad estadounidense de Seattle en 1999, contra la que se organizan manifestaciones y concentraciones alrededor de las cuales se crea un sitio web en el que se deposita información y que se convierte en un verdadero nodo de comunicaciones para los manifestantes -llega a obtener dos millones de visitas en 1999, según Nicholas W. Jankowsky y Marieke Jansen (2003: 3)- lo cual es una gran repercusión para la época.

Entre sus principios fundacionales está la capacidad de todos sus miembros de crear, distribuir o filtrar sus propias noticias, pero también se establecen una serie de normas editoriales. Algunas de ellas contienen recomendaciones sobre los artículos, que deben ser investigaciones a largo plazo, crónicas de testigo directo, análisis de medios, reportajes sobre injusticias, artículos que traten sobre colectivos invisibilizados, o artículos que traten proyectos de justicia social (Jankowsky y Jansen, 2003: 8).

Respecto a la versión ateniense de Indymedia, Milioni establece en su trabajo las funciones que tienen las diversas informaciones y contenidos depositados en la web de Indymedia Atenas dentro de su comunidad de lectores y redactores. De esta forma, extrae una serie de funciones que realiza este medio alternativo que tienen mucha relación con los medios tradicionales, ya sean de tipo ejemplar, cuando muestran la oposición con las formas de funcionar de los medios convencionales, (Milioni, 2009: 419); competitivo, con la creación de una agenda aparte de la de los medios convencionales; o suplementario, cuando tratan de complementar informaciones dadas en grandes medios o revisar su objetividad y autenticidad.

Como conclusión, la investigadora griega destaca tres rasgos fundamentales en Indymedia Atenas: información determinada por la acción individual, relativa a la creación de una opinión pública al margen de los medios convencionales; interactividad, relativa a la comunicación nacida a raíz de las propias noticias y en los comentarios; y acción en red deslocali- 
zada, que pone en valor la noción de este medio como espacio de colaboración. A raíz de este último rasgo, el que permite la comunicación entre personas no conectadas entre sí pero pertenecientes a una misma sociedad civil se produce para Milioni "una dirección a partir de la cual volver a pensar, con cautela pero con optimismo, el significado de la esfera pública contemporánea” (Milioni, 2009: 427).

El trabajo de Juris, fundamentado en testimonios de activistas de Barcelona y de coordinadores de la red en Australia, abunda sobre las características generales de la red, sus métodos de coordinación y toma de decisiones y apunta uno de los principales problemas de esta red: las decisiones de control editorial, que para algunos miembros deberían ser casi inexistentes, mientras que para otros resulta necesario algún tipo de intermediación en los contenidos "de acuerdo a ciertas pautas definidas por los objetivos de justicia social de la Red" (Juris, 2004: 173).

Este estudio enlaza con nuestro proyecto en tanto indaga en los contenidos que se producen en un medio alternativo y su capacidad de crear comunidad, de la misma forma que nosotros examinamos la producción de medios alternativos y convencionales como formas distintas -no necesariamente opuestas- de crear comunidades y de aportar nociones a la opinión pública, por medio de estrategias de encuadre o framing.

Por otra parte, en la historia reciente de España podemos registrar un antecedente de movilizaciones que se pueden relacionar con el 15-M en lo tocante al papel de los medios de comunicación alternativos, como es el caso de las manifestaciones que siguieron a los atentados del 11 de marzo de 2004 en Madrid, específicamente el día 13, jornada de reflexión de unas elecciones generales a nivel nacional en la que se produjeron varias concentraciones de protesta ante sedes del partido político gobernante en aquel momento, el Partido Popular.

Estas movilizaciones, producidas en una situación de desconcierto informativo respecto a la autoría del atentado -entre la línea oficial que apuntaba a la responsabilidad de la organización terrorista ETA y varios medios que relacionaban el suceso con el terrorismo islámico y la participación de España en la Guerra de Irak- y en un contexto electoral, mostraron un ejemplo de "ruptura del bloqueo informativo" protagonizado por medios alternativos, según aseguran Sara López Martín y Gustavo Roig 
«Tratamiento periodístico del Movimiento 15-M en medios de comunicación alternativos...»

Domínguez en uno de los capítulos del libro 13-M Multitudes online, editado por Víctor Sampedro.

López Martín y Roig Domínguez analizan los sitios web de los "nodos" de Indymedia en Madrid y Barcelona junto a otros dos medios alternativos en la Red como La Haine y Nodo 50, y concluyen que son estos medios "los que rompen el bloqueo informativo generado tras los atentados del 11-M" (López Martín y Roig Domínguez, 2005: 47) y se erigen como nuevos "referentes de opinión" a una determinada escala ante el desconcierto creado por las informaciones procedentes de la prensa tradicional.

Salvando las lógicas distancias entre ambos acontecimientos, entendemos este antecedente como otra muestra de la relevancia que, en momentos de gran actividad social, obtiene el discurso y la forma de enfocar las informaciones de medios alternativos que ofrecen una alternativa a la visión mediática tradicional y muestran formas distintas de encarar la realidad, una inquietud que está en la base de los objetivos de nuestro estudio.

\section{Metodología}

El procedimiento empleado para realizar el estudio ha constado de dos fases diferenciadas: un primer paso cuantitativo, en el que se han rastreado las versiones digitales de los cuatro medios objeto de estudio para extraer los artículos objeto de análisis y un segundo período de análisis cualitativo, en el que los artículos seleccionados han sido tratados por medio de una ficha de análisis en la que se han tenido en cuenta aspectos pertenecientes al análisis de contenido y a las estrategias de encuadre o framing elaboradas por cada medio, con la intención de sacar conclusiones sobre el comportamiento de cada grupo de medios.

La fase cuantitativa se hizo por medio de las secciones de "Archivo" en el caso de Público y El País, rastreando día por día las noticias relacionadas de alguna forma con el Movimiento 15-M que estuvieran fechadas entre el 15 de mayo y el 31 de ese mes; un proceso que en el caso de Periodismo Humano y Diagonal se vio facilitado por la existencia en ambos medios de una sección especial dedicada a la cobertura del acontecimiento, que servía de repositorio de informaciones. 
«Tratamiento periodístico del Movimiento 15-M en medios de comunicación alternativos...»

Los artículos encontrados en los archivos de los medios digitales fueron contrastados a través de búsquedas selectivas en los motores que ofrecían los propios medios, lo que permitió localizar artículos que no estaban en las secciones concretas pero que también pertenecían al espectro temporal que se trataba. En algunas ocasiones, incluso hubo que incorporar artículos nuevos durante las primeras etapas del análisis cualitativo.

El proceso final dio lugar a una ingente cantidad de artículos, 461 piezas periodísticas repartidas de forma bastante desigual entre los medios: 128 artículos de El País, 198 de Público, 40 de Periodismo Humano y 95 de Diagonal, que fueron clasificados por días y almacenados en formato de documento PDF por orden cronológico, para proceder después a su análisis, en el que se les incorporaba en el nombre de archivo un código alfanumérico que permitía su clasificación en la base de datos.

A partir del material recogido, se procedió a aplicar la técnica del análisis de contenido, una metodología académica de larga tradición orientada a descubrir y determinar los aspectos significativos del mensaje y especialmente establecer "su contenido ideopolítico y su intencionalidad" (Páez Warton, 1989), que tiene en su aplicación al periodismo numerosos ejemplos, como el del estudioso estadounidense Harold Laswell, que lo aplicó ya a la prensa para tratar el uso de la propaganda durante la Primera Guerra Mundial (Propaganda techniques in the World War, 1927, citado por Andreu Abela, 200 1: 5).

El sociólogo estadounidense Bernard Berelson es quien elabora un primer manual de análisis de contenido en investigaciones sobre comunicación (Content Analysis in Communication Research) en el que la describe como una técnica para "la descripción objetiva, sistemática y cuantitativa del contenido manifiesto de la comunicación" (Berelson, 1952, en Andreu Abela 2001:2), siendo la parte cuantitativa de este procedimiento la más problemática en el ámbito de la comunicación, ya que para muchos autores se trata de una técnica que es incapaz de extraer elementos muy necesarios en comunicación, como para el alemán Jürgen Ritsert, que critica la incapacidad del análisis de contenido para extraer estructuras de sentido latente procedentes del contexto, por ejemplo.

Para saltar este problema del análisis de contenido en comunicación son especialmente importantes las categorías de análisis, que para Philipp Mayring deben estar cuidadosamente establecidas y revisadas, en un análi- 
«Tratamiento periodístico del Movimiento 15-M en medios de comunicación alternativos...»

sis que "evite la cuantificación precipitada" (Mayring 2000, citado en Kohlbacher, 2005).

Dentro de este análisis de contenido se utilizó el enfoque de la estrategia de encuadre o framing, una teoría para el estudio de los medios que da un paso más respecto a la idea de agenda setting acuñada por McCombs y Shaw (1972) y la relaciona con los marcos de interpretación que se utilizan en el ámbito de la psicología. Desde ese ámbito psicológico, autores como Bateson o Goffman definen los encuadres como construcciones colectivas para entender el mundo en las que caben las estructuras mentales individuales.

Para el norteamericano Robert Entman, uno de los pensadores que traslada este concepto al estudio de los medios de comunicación, el concepto de encuadre permite "describir consistentemente el poder de un texto comunicativo" influencia sobre la consciencia humana es ejercida a través de la transferencia de información" (Entman, 1993: 51). Se trata de construcciones que, según este autor, tienen más valor por lo que omiten que por lo que muestran e influyen de manera más radical sobre los asuntos que quedan fuera del marco que sobre aquellos que entran dentro del encuadre.

En este ámbito, también resulta interesante la visión crítica de Paul D’Angelo (2002), que pone en valor la diferencia entre aproximarse a los encuadres entendiéndolos como parte del trabajo del periodista o contemplarlos como muestra de un proceso de hegemonía mediática, en el que los encuadres responden a la necesidad de unas élites políticas y/o económicas detrás de la prensa que tratan de ocultar unos temas y privilegiar otros.

El proceso de analizar los artículos requirió el uso de varias nociones del encuadre, de manera que en la ficha de análisis final entraron tanto categorías inspiradas en las propuestas de autores como Entman, del cual extrajimos una distinción básica de encuadre con cuatro opciones (Definición del problema / Atribución de responsabilidad / Valoración moral / Recomendación de tratamiento), pero también de otros estudiosos.

La estrategia de cinco tipologías de encuadre Holli A. Semetko y Patti M. Valkenbourg, que habían utilizado en un estudio sobre la prensa y la televisión europeas (2000), también fue empleada, en este caso para los

\footnotetext{
${ }^{1}$ Traducción propia del texto original.
}

Mediaciones Sociales, № 12, 2013, pp. 160-187. ISSN electrónico: 1989-0494. DOI: http://dx.doi.org/10.5209/rev_MESO.2013.n12.45267 
«Tratamiento periodístico del Movimiento 15-M en medios de comunicación alternativos...»

titulares, por su capacidad de ofrecer más alternativas a la hora de definir los marcos por medio de cinco categorías (Conflicto / Interés humano / Consecuencias económicas / Moralidad / Responsabilidad).

Además, también se agregaron las categorías propuestas según grado de generalización que elabora el investigador indio Shanto Yvengar (1991, en Rabadán, 2010), que por medio de una división simple (Temático / Episódico), ofrecía una distinción fundamental, la de si el artículo estaba inscrito en un trabajo amplio de investigación y contraste o si se trataba de una cobertura puntual y basada en el acontecimiento concreto.

A estas categorías extraídas de autores previos se agregaron otras muchas de carácter cualitativo y elaboración propia, hasta llegar a confor-

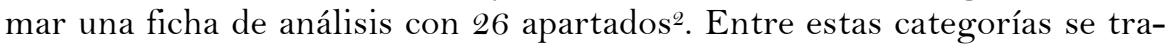
taba la tipología de artículo (Crónica / Reportaje / Noticia / Entrevista / Columna de opinión / Editorial), la sección en la que estaba situado, el formato del titular (Descriptivo / Interpretativo / Declaración entrecomillada) y una serie de categorías basadas en la contabilización de las fuentes utilizadas y los actores aludidos en el texto. Además, se incluyó otra categoría de análisis relativa a lo que se denominó como "soportes ideológicos o inspiradores del movimiento", personas destacadas como soporte moral o intelectual de las movilizaciones por parte de los acampados o de los medios periodísticos -caso de pensadores como el francés Stéphane Hessel o el español José Luis Sampedro-.

Una de ellas es la denominada como "rasgos atribuidos al 15-M", en la que se establecieron una serie de categorías previstas en función de las afirmaciones que se producían en los artículos respecto referencia al comportamiento de los acampados y al carácter de las asambleas, a las valoraciones externas de los acampados y manifestantes mostradas en el texto, a datos cuantitativos de participación o a la repercusión de los comportamientos de los acampados en terceros, agrupadas en tres grupos (positivo/negativo/neutro) en función de la valorización que se producía acerca de las actitudes pertenecientes o relativas al movimiento y a sus diversos actores. Entre ellas se incluían nociones positivas como "discusión política”, "organización innovadora", "representatividad", "propuestas pertinentes”; neutras, como "participación”, “enumeración de propuestas”; o negati-

\footnotetext{
${ }^{2}$ Ver Anexo 1.
}

Mediaciones Sociales, № 12, 2013, pp. 160-187. ISSN electrónico: 1989-0494. DOI: http://dx.doi.org/10.5209/rev_MESO.2013.n12.45267 
«Tratamiento periodístico del Movimiento 15-M en medios de comunicación alternativos...»

vas, como "instrumentalización política", "problemas del método asambleario", "violencia policial" o "violencia 15-M".

Todas estas categorías fueron instrumentalizadas en la parte cualitativa a partir de la contabilización de la aparición de cada una de ellas respecto del total de los artículos de cada medio, obteniendo un porcentaje que mostraba su grado de aparición respecto al número de artículos analizados de su cabecera. Esta decisión se toma al constatar que el número de artículos de cada medio es muy desigual, hecho que viene dado por la disparidad de estructuras (El País y Público son medios con mayor número de redactores y recursos que Periodismo Humano y Diagonal), de manera que el uso de cifras relativas era la única manera de realizar un análisis comparable.

De forma complementaria a la elaboración de categorías, se realizaron entrevistas personales a periodistas de los cuatro medios objeto de análisis y relacionados con la cobertura del Movimiento 15-M, con la intención de comprender cómo habían sido los enfoques informativos desde el quehacer periodístico de cada uno de los medios. Los encuentros fueron con periodistas y reporteros que en los meses del estudio formaban parte de los medios analizados, como Carmen Pérez-Lanzac (El País), Héctor Juanatey (Público), Juan Luis Sánchez (Periodismo Humano) y Héctor Rojo (Diagonal).

\section{ANÁLISIS}

Los resultados cuantitativos de la investigación ofrecen una primera clasificación intuitiva en función del número de artículos publicados, donde se destacan Público, con 198 piezas, y El País, con 128. Su idiosincrasia como organización periodística profesional y su apoyo en las agencias de noticias -especialmente notable en el caso de Público, con más de un 25\% de su cobertura en base a noticias de agencia- les permite tener este mayor número de informaciones, frente a las 95 de Diagonal y las 40 de Periodismo Humano.

Mucho más relevante acerca de la incidencia de la cobertura resulta el dato del número de artículos publicados por cada medio de forma diaria. En este caso, constatamos la importancia de las jornadas previas a las elecciones municipales y autonómicas del 22 de mayo del 2011 , ya que fueron los días 19, 20 y 21 los que suscitaron mayo número de artículos en los cuatro medios. Otro punto importante de interés estuvo en torno al día 28 
«Tratamiento periodístico del Movimiento 15-M en medios de comunicación alternativos...»

de mayo, en el que se produjeron choques entre policía y manifestantes con la orden de desalojo de la acampada de Barcelona.

Tabla 1: Distribución cronológica de artículos

\begin{tabular}{|cccccc|}
\hline FECHA & El País & Público & P. Humano & Diagonal & TOTAL \\
\hline $15 / 05 / 2011$ & 3 & 3 & 0 & 1 & 7 \\
\hline $16 / 05 / 2011$ & 2 & 5 & 1 & 4 & 12 \\
\hline $17 / 05 / 2011$ & 7 & 18 & 2 & 7 & 34 \\
\hline $18 / 05 / 2011$ & 5 & 19 & 1 & 4 & 29 \\
\hline $19 / 05 / 2011$ & 13 & 30 & 3 & 14 & 60 \\
\hline $20 / 05 / 2011$ & 16 & 38 & 6 & 16 & 76 \\
\hline $21 / 05 / 2011$ & 10 & 25 & 4 & 20 & 59 \\
\hline $22 / 05 / 2011$ & 8 & 4 & 3 & 7 & 22 \\
\hline $23 / 05 / 2011$ & 7 & 4 & 4 & 3 & 18 \\
\hline $24 / 05 / 2011$ & 3 & 4 & 3 & 1 & 11 \\
\hline $25 / 05 / 2011$ & 7 & 4 & 3 & 2 & 16 \\
\hline $26 / 05 / 2011$ & 5 & 5 & 1 & 3 & 14 \\
\hline $27 / 05 / 2011$ & 9 & 11 & 3 & 6 & 29 \\
\hline $28 / 05 / 2011$ & 16 & 6 & 1 & 0 & 23 \\
\hline $29 / 05 / 2011$ & 7 & 9 & 1 & 0 & 17 \\
\hline $30 / 05 / 2011$ & 5 & 7 & 3 & 4 & 19 \\
\hline $31 / 05 / 2011$ & 5 & 6 & 1 & 3 & 15 \\
\hline TOTAL & 128 & 198 & 40 & 461 \\
\hline & & Fuente: Elaboración propia & & \\
\hline
\end{tabular}

Como ya indicamos en el apartado anterior, la gran diferencia en número absoluto de artículos en cada una de las publicaciones, fruto de sus diferentes estructuras profesionales (número de periodistas, disponibilidad de recursos, etc.), provoca que las categorías se analicen a partir de números relativos, que son porcentajes de aparición de cada categoría dentro del total de artículos de cada medio. 
«Tratamiento periodístico del Movimiento 15-M en medios de comunicación alternativos...»

La aplicación de las categorías de Semetko y Valkenburg a los titulares (Conflicto / Interés humano / Consecuencias económicas / Moralidad / Responsabilidad), permite apreciar un predominio de los artículos de conflicto e interés humano. Entre los medios, destacan Diagonal y Público como los más asiduos al uso del framing conflictivo, mientras que Periodismo Humano ofrece el enfoque de interés humano en casi la mitad de sus artículos (47,5 por ciento de sus artículos).

Las informaciones analizadas a partir de las clasificaciones de Entman (Definición del problema / Atribución de responsabilidad / Valoración moral / Recomendación de tratamiento), mostraron a Diagonal como el más interesado en la definición del problema (más del 85 por ciento de sus informaciones fueron categorizadas de esa manera), mientras que Público fue el más interesado en las atribuciones de personalidad (más del 60\%). En la recomendación de tratamiento, o la búsqueda de viabilidad futura al 15-M, se destacó Periodismo Humano, con uno de cada diez artículos basados en ese tema.

Tabla 2: Encuadre de artículos según tipología de Entman

\begin{tabular}{|c|ccccc|}
\hline Encuadre del artículo & \multicolumn{5}{|c|}{ Porcentaje de artículos } \\
\hline & El País & Público & P. Humano & Diagonal & TOTAL \\
\hline Definición del problema & $40,60 \%$ & $56,10 \%$ & $57,50 \%$ & $85,30 \%$ & $57,90 \%$ \\
\hline Atribución de responsabilidad & $42,20 \%$ & $61,60 \%$ & $45 \%$ & $48,40 \%$ & $52,10 \%$ \\
\hline Valoración moral & $22,70 \%$ & $19,70 \%$ & $22,50 \%$ & $21,10 \%$ & $21 \%$ \\
\hline Recomendación de tratamiento & $5,50 \%$ & $4,50 \%$ & $10 \%$ & $4,20 \%$ & $5,20 \%$ \\
\hline \multicolumn{5}{c}{ Fuente: elaboración propia } \\
\hline
\end{tabular}

En el ámbito de la temporalidad, para el que se utilizó la división Temáticos/Episódicos propuesta por Yvengar predominó un seguimiento temático, es decir, tomado como un acontecimiento en desarrollo y no aislado, aunque el porcentaje de tratamiento episódico es sensiblemente más alto en medios como El País y Público, que ubicaron en este tipo entre el 30 y el 40 por ciento de sus artículos. 
«Tratamiento periodístico del Movimiento 15-M en medios de comunicación alternativos...»

Tabla 3: Encuadre temporal de los artículos según tipología de Yvengar

\begin{tabular}{|c|c|c|c|c|c|}
\hline Enfoque temporal & Porcentaje de artículos \\
\hline & El País & Público & P. Humano & Diagonal & TOTAL \\
\hline Temático & $71,90 \%$ & $64,10 \%$ & $90,00 \%$ & $89,50 \%$ & $73,60 \%$ \\
\hline Episódico & $27,30 \%$ & $35,90 \%$ & $2,50 \%$ & $9,50 \%$ & $25,20 \%$ \\
\hline \multicolumn{7}{|c|}{ Fuente: elaboración propia } \\
\hline
\end{tabular}

Resalta, respecto a los titulares periodísticos, el predominio del titular más interpretativo frente al descriptivo, mientras que se observa mayor número de artículos con declaraciones entrecomilladas -el formato más habitual para la declaración política, también utilizado en entrevistas- en su titular en medios como Público (un 27,3 por ciento de sus artículos se iniciaban con estas declaraciones), mientras que en Diagonal tan solo un 8,4 por ciento de los artículos utilizan este tipo de titulares.

Asimismo, en el ámbito de los géneros periodísticos hay que destacar el uso residual de la entrevista, menor del 5 por ciento del total de las piezas analizadas, lo que permite caracterizar la respuesta de los medios en los primeros 15 días del Movimiento 15-M como de muy limitada a la hora de apoyarse en géneros de mayor elaboración como el de la entrevista -de los protagonistas o de otras personalidades- para explicar el fenómeno.

También dentro de los usos periodísticos, el trabajo con fuentes ofrece una clara dicotomía en la diferencia entre medios alternativos y convencionales, ya que estos últimos ofrecen un protagonismo a fuentes gubernamentales de los tres niveles (estatal-autonómico-municipal) y a los tres partidos más mencionados (PP-PSOE-IU) que es inexistente en el caso de los alternativos. 
«Tratamiento periodístico del Movimiento 15-M en medios de comunicación alternativos...»

Tabla 4: Uso de partidos políticos como fuente

\begin{tabular}{|c|c|c|c|c|c|}
\hline Fuente política & Medios & \\
\hline & E1 País & Público & P. Humano & Diagonal & TOTAL \\
\hline PP & $7 \%$ & $8,10 \%$ & $0 \%$ & $0 \%$ & $5,40 \%$ \\
\hline PSOE & $7 \%$ & $7,60 \%$ & $0 \%$ & $0 \%$ & $5,20 \%$ \\
\hline IU & $8,60 \%$ & $13,60 \%$ & $0 \%$ & $0 \%$ & $8,20 \%$ \\
\hline Otros partidos & $0,80 \%$ & $2,50 \%$ & $0 \%$ & $0 \%$ & $1,30 \%$ \\
\hline \multicolumn{7}{|c|}{ Fuente: elaboración propia } \\
\hline
\end{tabular}

En el ámbito de las fuentes, coinciden ambos tipos de medio en el uso de la información de activistas y portavoces del 15-M (en el que se destaca especialmente Periodismo Humano), mientras que la voz de las asociaciones sociales está más destacada en los alternativos que en los convencionales. En los actores mencionados también se produce esta distinción, que solo ofrece una variación en el caso de la policía, mencionada asiduamente por ambos tipos de medio en porcentajes superiores al 30 por ciento de los artículos.

Las denominaciones a los manifestantes fueron estudiadas con intención de analizar cuáles fueron los nombres más utilizados para referirse a los manifestantes y si el tipo del medio determinada una manera de nombrar diferente. Este es el caso del apelativo "indignados", extraído del texto de Stéphane Hessel Indignaos, que en El País y Público supera el 35 por ciento de los artículos, mientras que no alcanza el 20 en Diagonal y Periodismo Humano, medios que se inclinaron más por denominaciones como “asambleas", especialmente en el caso de Diagonal, que se utiliza en más de la mitad de los artículos, además de designaciones más genéricas como la de "acampados" o "Movimiento 15-M", que aparecen de forma similar en ambos grupos de medios.

Respecto a los rasgos atribuidos al 15-M, estas categorías planteadas en función de los aspectos que potenciaban los artículos dentro de las diferentes coberturas del Movimiento 15-M, las más destacadas por su porcentaje de aparición en los textos son "participación" (40 por ciento de los artículos), "discusión política", "participación variada” y "organización 
«Tratamiento periodístico del Movimiento 15-M en medios de comunicación alternativos...»

innovadora" (con un porcentaje cercano al 30 por ciento), todas ellas agrupadas dentro de las categorías neutras o de carácter positivo; mientras que la más destacada dentro de las negativas fue "instrumentalización política" (14,5 por ciento), junto a otras como "problemas asamblearios", "violencia policial”, "perjuicios a vecinos" o "rechazo político", todas con porcentajes cercanos al 10 por ciento.

Tabla 5: Principales rasgos atribuidos al 15-M presentes en cada medio

\begin{tabular}{|c|c|c|c|c|c|}
\hline \multirow[t]{2}{*}{ Rasgos atribuidos al 15-M } & \multicolumn{3}{|c|}{ Porcentaje de artículos } & \multirow[b]{2}{*}{ Diagonal } & \multirow[b]{2}{*}{ TOTAL } \\
\hline & El País & Público & P. Humano & & \\
\hline (+) Discusión política & $34,40 \%$ & $26,30 \%$ & $42,50 \%$ & $36,80 \%$ & $32,10 \%$ \\
\hline$(+)$ Participación variada & $27,30 \%$ & $27,80 \%$ & $50,00 \%$ & $37,90 \%$ & $31,70 \%$ \\
\hline$(+)$ Organización innovadora & $28,10 \%$ & $21,20 \%$ & $42,50 \%$ & $40,00 \%$ & $28,90 \%$ \\
\hline (+) Representatividad & $7,80 \%$ & $23,70 \%$ & $7,50 \%$ & $9,50 \%$ & $15,00 \%$ \\
\hline$(+)$ Propuestas pertinentes & $18,00 \%$ & $10,10 \%$ & $7,50 \%$ & $3,20 \%$ & $10,60 \%$ \\
\hline (+) Internacionalización $15-\mathrm{M}$ & $2,30 \%$ & $10,10 \%$ & $15,00 \%$ & $14,70 \%$ & $9,30 \%$ \\
\hline (+) Pacifismo 15-M & $15,60 \%$ & $7,10 \%$ & $10,00 \%$ & $8,40 \%$ & $10,00 \%$ \\
\hline (N) Participación & $32,00 \%$ & $29,30 \%$ & $35,00 \%$ & $71,60 \%$ & $39,30 \%$ \\
\hline (N) Enumeración propuestas & $14,10 \%$ & $8,60 \%$ & $20,00 \%$ & $12,60 \%$ & $11,90 \%$ \\
\hline (-) Perjuicios vecinos, suciedad & $14,10 \%$ & $8,10 \%$ & $7,50 \%$ & $4,20 \%$ & $8,90 \%$ \\
\hline (-) Instrumentalización política & $10,20 \%$ & $24,70 \%$ & $0,00 \%$ & $5,30 \%$ & $14,50 \%$ \\
\hline (-) Problemas asamblearios & $16,40 \%$ & $9,60 \%$ & $12,50 \%$ & $4,20 \%$ & $10,60 \%$ \\
\hline (-) Dominación grupos & $1,60 \%$ & $1,50 \%$ & $0,00 \%$ & $0,00 \%$ & $1,10 \%$ \\
\hline (-) Perjuicios comerciantes & $11,70 \%$ & $2,50 \%$ & $5,00 \%$ & $0,00 \%$ & $4,80 \%$ \\
\hline (-) Violencia policía & $9,40 \%$ & $9,60 \%$ & $12,50 \%$ & $5,30 \%$ & $8,90 \%$ \\
\hline (-) Violencia 15-M & $7,00 \%$ & $2,50 \%$ & $2,50 \%$ & $0,00 \%$ & $3,30 \%$ \\
\hline (-) Rechazo medios & $3,10 \%$ & $4,00 \%$ & $2,50 \%$ & $6,30 \%$ & $4,10 \%$ \\
\hline (-) Rechazo políticos & $5,50 \%$ & $11,10 \%$ & $2,50 \%$ & $11,60 \%$ & $8,90 \%$ \\
\hline Leyenda: Rasgos & didos & $\begin{array}{l}\text { tivos } \\
\text { oracić }\end{array}$ & $\begin{array}{l}\text { eutros }(\mathrm{N} \\
\text { ppia }\end{array}$ & ativos & \\
\hline
\end{tabular}

Mediaciones Sociales, № 12, 2013, pp. 160-187. ISSN electrónico: 1989-0494. DOI: http://dx.doi.org/10.5209/rev_MESO.2013.n12.45267 
«Tratamiento periodístico del Movimiento 15-M en medios de comunicación alternativos...»

Respecto a estos conceptos, tomados por tipología de medio, encontramos un énfasis especial en los medios alternativos de la variedad en los participantes, la discusión política y la innovación en las organizaciones de las movilizaciones (con porcentajes cercanos al 50 por ciento de los artículos); mientras que los medios tradicionales destacan esos rasgos de forma más moderada (entre el 30 y el 20 por ciento), e incluyen otros debates como la instrumentalización política (24,7\% en Público).

Para concluir, conviene destacar notablemente entre los resultados uno relacionado con el trabajo multimedia de cada medio, en el que resulta muy interesante entender que, contra lo que podría dar a entender la amplitud de las organizaciones y la potencia de personal de los medios tradicionales, es uno alternativo, Periodismo Humano, el que más contenido audiovisual ha colocado en su portal de producción propia (16 vídeos), aunque en el cómputo general de vídeos tenga a Público por encima, cuyos contenidos multimedia fueron esencialmente (24 de 36) extraídos de otras agencias audiovisuales.

\section{Conclusiones}

El estudio de las coberturas de medios convencionales y alternativos en el contexto del Movimiento 15-M ha dado lugar a una serie de conclusiones que ofrecen varias claves sobre las cuales comprender la división y las distintas formas de entender el desempeño del periodismo desde dos tipos de estructura mediática distintos.

Respecto a la primera hipótesis, que auguraba una mayor atención a los temas de alcance social en el caso de los medios alternativos, no podemos considerarla refutada por completo ya que el tratamiento del 15-M ha tenido en términos cuantitativos una gran repercusión también en los medios tradicionales, que ya de por sí tienen mayor capacidad profesional para generar información mayor en volumen que los alternativos.

Sin embargo, en las hipótesis también augurábamos una mayor atención al ámbito político y declarativo, que sí se ha demostrado más presente en medios de carácter convencional, como Público -algo que fue reconocido por miembros del propio medio, como el exredactor de público.es Héctor Juanatey-, donde superaba una cuarta parte de las informaciones, frente a 
«Tratamiento periodístico del Movimiento 15-M en medios de comunicación alternativos...»

otros como Diagonal en el que tan solo se podía encontrar en algo menos del diez por ciento de las noticias.

Esta percepción fue corroborada desde el punto de vista de los medios alternativos, Juan Luis Sánchez, de Periodismo Humano:

“Al principio... [los grandes medios] no entendían lo que estaba pasando. Y como no lo entendían (...) no intentaron comprenderlo, porque no entraba dentro de su clave de codificación de las cosas: o algo es de izquierda o de derecha, o beneficia a uno o a otro" (Sánchez, Juan Luis. Entrevista telefónica el 1 de septiembre de 2011 ).

Respaldan también esta hipótesis los datos sobre la utilización de partidos políticos como fuente, presentes en los medios convencionales pero prácticamente inéditos en Periodismo Humano y Diagonal.

En cuanto a la noción que apuntaba a la cobertura más local por parte de los medios alternativos, su funcionamiento en Internet ha desvirtuado un tanto esta hipótesis, para ofrecernos un panorama distinto: medios como Diagonal, con una estructura cercana a lo informal, ofrecieron más de un tercio de sus noticias sobre acampadas que no eran la madrileña o la barcelonesa, aprovechando su red de colaboradores sin ánimo de lucro que opera desde varios puntos del territorio, según nos explicó Héctor Rojo, redactor de Diagonal. Sin embargo, las grandes organizaciones periodísticas como El País o Público condensaron su cobertura informativa prácticamente en Madrid y Barcelona.

Respecto a los elementos del encuadre o framing, encontramos un predominio en general en los titulares de las informaciones del encuadre de conflicto, si bien Periodismo Humano se ha destacado en el marco de interés humano, hallado en piezas periodísticas más extensas por lo general -más de 1.000 palabras por artículo, mientras que El País y Público se han situado en la órbita de las 700 y Diagonal en 500-.

En las categorías aplicadas al conjunto de los textos, los enfoques de definición de conflicto y atribución de responsabilidad han sido predominantes en todos los medios, motivados por las necesidades básicas a la hora de enfrentar un fenómeno informativamente desconocido para los medios, que trataron de posicionar a los actores en las movilizaciones y de buscar responsabilidades de lo sucedido. Sin embargo, se echa de menos 
«Tratamiento periodístico del Movimiento 15-M en medios de comunicación alternativos...»

más presencia del encuadre de recomendación de tratamiento, que tan solo tiene una presencia reseñable en el caso de Periodismo Humano, en el que aparece en un 10 por ciento de los artículos.

Otro de los datos que permiten distinguir diferencias entre tipologías de medio viene dado por la clasificación temporal en los enfoques a través de las categorías de Yvengar, en las cuales se aprecia un mayoritario uso del framing temático a nivel general, lo que permite entender que en todos los medios se informó del Movimiento 15-M describiéndolo como un acontecimiento no aislado, sino en progresión, y de forma exhaustiva en la que se ofreció contexto. Sin embargo, si bien el ámbito temático aparece por igual en todos los medios, se encuentra una mayor presencia del enfoque episódico en El País y Público, con porcentajes del 27 y el 35 por ciento, respectivamente.

También se refleja una diferencia clave entre convencionales y alternativos, en la atención a partidos políticos como fuente y como sujeto aludido en las piezas informativas. Tanto El País como Público recurrieron a fuentes políticas en porcentajes cercanos al 8 por ciento, a diferencia de la ausencia total que esas mismas fuentes tuvieron en Público y Periodismo Humano. Si nos referimos a los actores mencionados, formaciones políticas como el Partido Popular aparecen en más del 20 por ciento de los artículos en medios convencionales, con el PSOE en porcentajes algo inferiores (7 por ciento y 17 por ciento); sin embargo, sus menciones en medios alternativos no alcanzan en ninguno de ellos el 10 por ciento de los artículos.

Como confirmaba Héctor Juanatey, periodista de Público, en muchos medios convencionales había "mucha incertidumbre al principio sobre quién estaba detrás” del 15-M (Juanatey, Héctor. Entrevista telefónica el 11 de septiembre de 2012), lo cual provocó en medios convencionales la tendencia a acudir a fuentes políticas en búsqueda de respuestas, con la tensión añadida de una atmósfera electoral. "Las elecciones daban para mucho escrito sobre a quién perjudicaban, si a la izquierda o a otros, cuando en realidad ellos no estaban allí para perjudicar a nadie”, insistió Juanatey (ibid).

En la forma de denominar a los participantes en el Movimiento 15-M también podemos resaltar el rechazo de los medios alternativos al apelativo "indignados", profusamente utilizado por los convencionales, y la opción por parte de Periodismo Humano y Público por denominaciones que enfatizaban claramente el carácter democrático de los acampados como 
«Tratamiento periodístico del Movimiento 15-M en medios de comunicación alternativos...»

"asambleas", además de definiciones neutras como "acampados", que si bien fue implementada también por medios tradicionales, añadida a la consabida "Movimiento 15-M", empleada por igual en todos los tipos de medio.

Respecto a las valoraciones inherentes en los artículos, que han sido analizadas a partir de las rasgos atribuidos, se ha percibido una predilección por parte de los medios alternativos respecto a los aspectos más democráticos, innovadores y políticos en el sentido de deliberativos del 15M, mientras que la instrumentalización política ha estado más presente en medios como Público y en menor medida en El País.

De esta forma, encontramos dos coberturas bastante diferenciadas ante un hecho inesperado como fueron las manifestaciones del 15 de mayo, en la que medios tanto alternativos como convencionales encontraron un acontecimiento que alteraba sus agendas habituales, pese a que medios como Diagonal ya hubieran incorporado previamente a los convocantes de las manifestaciones, ya que, como nos explicaba Héctor Rojo, redactor de Diagonal, ellos ya contaban con "fuentes y contactos" en estos movimientos sociales. "Todas las manifestaciones no salen de la nada, sino que están perfectamente organizadas por gente que está detrás, que nosotros conocemos e incluso que formamos parte en ocasiones", aseguró Rojo (Rojo, Héctor. Entrevista personal el 2 de septiembre de 2011).

Si los medios alternativos trataron de organizar sus coberturas aprovechando su cercanía a las asociaciones sociales, ambos tipos de medio recurrieron habitualmente a los acampados y sus portavoces, mientras que los medios convencionales mostraron su dependencia de los mensajes de los partidos políticos, obligados a reaccionar ante un movimiento social inesperado, en un escenario electoral que también se vio reflejado en el incremento de la cobertura en las jornadas previas a los comicios.

La independencia de los medios alternativos respecto de la política no se vio reflejada en una mayor profundidad en la cobertura -como demuestra la ausencia, por ejemplo, de entrevistas en ambos grupos- aunque sí de un trabajo más prospectivo, con un enfoque más amplio que el habitual de conflicto, como en el caso de Periodismo Humano con el framing de interés humano y la mayor longitud de sus noticias respecto a la media. También la cobertura de Periodismo Humano fue relevante en torno al trabajo con contenido multimedia, ya que su trabajo audiovisual de producción propia fue más intensivo que el de los dos medios convencionales, que sin embar- 
«Tratamiento periodístico del Movimiento 15-M en medios de comunicación alternativos...»

go contaban con más posibilidades técnicas para ofrecer imágenes propias del acontecimiento.

De esta forma, podemos concluir que las prácticas periodísticas de medios convencionales y alternativos durante el Movimiento 15-M ofrecieron dos tipos de cobertura ciertamente diferenciada ante un mismo hecho al que, si bien dirigieron una gran atención en ambos casos, fue encarado en el caso de los alternativos desde una perspectiva más cercana a los protagonistas y con una mayor profundidad, que en el caso de los medios convencionales se vio lastrada por rutinas periodísticas basadas en la información partidista, los despachos de agencia y la falta de innovación en contenidos periodísticos.

\section{Bibliografía}

Andreu Abela, J. (2001): Técnicas de Análisis de Contenido: una revisión actualizada. Sevilla: Fundación Pública Andaluza Centro de Estudios Andaluces, Junta de Andalucía.

AtTon, C. (2002): Alternative media. London: SAGE Publications.

Atton, C. y Hamilton, J.F. (2008): Alternative journalism. London: SAGE Publications.

D’Angelo, P. (2002): "News Framing as a Multiparadigmatic Research Program: A Response to Entman”, Journal of Communication, 54:4, pp. 870-88. New York: Oxford University Press.

Downing, J.; Mohammadi, A.; Sreberny-Mohammadi, A. (1990): Questioning the media. A critical introduction. Newbury, California: SAGE Publications.

ENTMAN, R.(1993). "Framing: Toward clarification of a fractured paradigm", Journal of Communication, 43: 4, pp. 51-58. New York: Oxford University Press.

GaLÁn, J. (2008): "Concepto y evolución de la empresa informativa”, en AGUADO, G.; Galán, J.; Fernández-BEaumont, J.; GarCía, L.J. (2008): Organización y gestión de la empresa informativa. Madrid: Síntesis.

Gomis, L. (1991): Teoría del Periodismo. Cómo se forma el presente. Barcelona: Paidós Comunicación.

Mediaciones Sociales, № 12, 2013, pp. 160-187. ISSN electrónico: 1989-0494.

DOI: http://dx.doi.org/10.5209/rev_MESO.2013.n12.45267 
JANKOWSKI, N.W. y JANSEN, M. (2003): "Indymedia: Exploration of an Alternative Internet-based Source of Movement News”, Conferencia Digital News, Social Change and Globalization. Impartida en la Hong Kong Baptist University los días 11 y 12 de diciembre de 2003.

JURIS, J. (2004): “Indymedia: de la Contra-Información a la Utopía Informacional", en MArí SÁez, V. (cood.), La Red es de todos. Cuando los Movimientos Sociales se apropian de la Red. Madrid: Editorial Popular.

KAPLÚN, M. (1985): El comunicador popular. Quito: Ciespal.

Kohlbacher, F. (2006): "The Use of Qualitative Content Analysis in Case Study Research”. Forum: Qualitative Social Research Sozialforschung, vol 7, num. 1, art, 21. Berlín: Institute for Qualitative Research and the Center for Digital Systems.

MC Combs, M. Y SHAU, D.L. (1972): "The Agenda-Setting Functions of the Mass Media”. Public Opinion Quaterly, vol. 36, pp. 176-187. New York: Oxford University Press.

Milioni, D.L. (2009): "Probing the online counterpublic sphere: the case of Indymedia Athens”. Media, Culture Eீ Society 31, pp. 409-431. Los Angeles, London, New Delhi and Singapore: SAGE Publications.

Monzón, C. (2006): “Opinión pública, comunicación y política”. Madrid: Editorial Tecnos.

PÁEZ Warton, J. (s/f): "El análisis de contenido".

URL: http://www.borrones.net/investigacion/contenido.pdf

Consultado: 12 de julio de 2013.

RABADÁN, P. (2010): "Nuevas vías para el estudio del framing periodístico. La noción de estrategia de encuadre”. Estudios sobre el mensaje periodístico, 2010: 16, pp. 235-258. Madrid: Universidad Complutense de Madrid.

Rodríguez, C. (2001): Fissures in the Mediascape: An International Study of Citizens' Media. Cresskill, NJ: Hampton Press. Citado en AtTon, C. y Hamilton, J.F. (2008): Alternative journalism. London: SAGE Publications.

Semetko, H.A. y Valkenburg, P.M. (2000): "Framing European Politics: A Content Analysis of Press and Television News". Journal of Communication, 50:2, pp. 93-109. New York: Oxford University Press. 


\section{PARA CITAR ESTE TRABAJO EN BIBLIOGRAFÍAS:}

MORENO RAMOS, Miguel Ángel (2013): “Tratamiento periodístico del Movimiento $15-\mathrm{M}$ en los medios alternativos y convencionales", Mediaciones Sociales. Revista de Ciencias Sociales y de la Comunicación, $\mathrm{n}^{\mathrm{o}}$ 12, pp. 160-187. DOI: http://dx.doi.org/10.5209/rev_MESO.2013.n12.45267

\section{${ }^{(*)}$ El autor}

Miguel Ángel Moreno Ramos (Madrid, 1988) es licenciado en Periodismo y Comunicación Audiovisual por la Universidad Carlos III de Madrid, además de contar con estudios en Información y Documentación, también en esta universidad. Ha realizado esta investigación en el marco de su Proyecto Fin de Carrera, bajo la dirección de Alejandro Barranquero Carretero.

RECIBIDO: 13 de julio de 2012.

ACEPTADO: 23 de enero de 2013. 